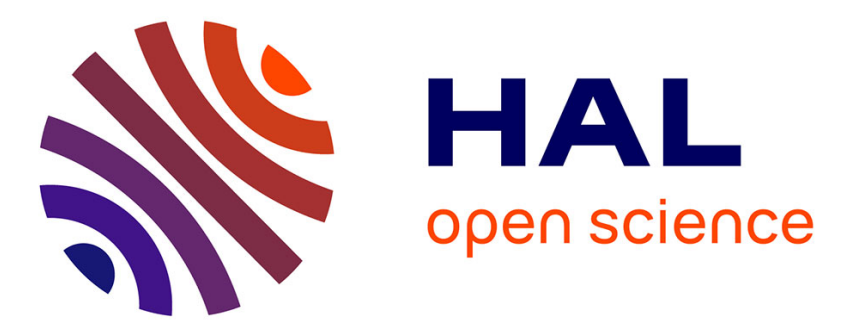

\title{
La place des filles dans l'enseignement supérieur scientifique en Chine: un pas en avant, deux pas en arrière
}

Christine Fontanini, Qingyu Wu

\section{- To cite this version:}

Christine Fontanini, Qingyu Wu. La place des filles dans l'enseignement supérieur scientifique en Chine: un pas en avant, deux pas en arrière. Les Sciences de l'éducation pour l'ère nouvelle: revue internationale, 2009, 2009 (4). hal-02943252

\section{HAL Id: hal-02943252 \\ https://hal.univ-lorraine.fr/hal-02943252}

Submitted on 18 Sep 2020

HAL is a multi-disciplinary open access archive for the deposit and dissemination of scientific research documents, whether they are published or not. The documents may come from teaching and research institutions in France or abroad, or from public or private research centers.
L'archive ouverte pluridisciplinaire HAL, est destinée au dépôt et à la diffusion de documents scientifiques de niveau recherche, publiés ou non, émanant des établissements d'enseignement et de recherche français ou étrangers, des laboratoires publics ou privés. 


\title{
La place des filles dans l'enseignement supérieur scientifique en Chine : un pas en avant, deux pas en arrière
}

\author{
Christine FonTANINI* et Qingyu Wu**
}

Résumé : Depuis dix ans, le nombre d'étudiants et d'étudiantes augmente considérablement en Chine. Pour autant, cette "massification» de l'enseignement supérieur n'entraîne pas, bien au contraire, une égalité entre les filles et les garçons dans l'accès aux différentes filières universitaires. Ainsi, on constate qu'avec l'évolution politique du système communiste, prônant une égalité entre les deux sexes dans la sphère professionnelle et avec l'ouverture de la Chine à l'économie de marché, les femmes et les hommes tendent à faire des choix d'études et professionnels semblables à leurs homologues du monde occidental, à savoir une orientation massive des filles vers les filières littéraires, sciences humaines et sociales... et les garçons, vers les sciences et techniques.

Mots-clés : Sciences. Chine. Orientation. Genre.

${ }^{*}$ Christine Fontanini, Maître de Conférenes, CREAD, Université Rennes 2.

${ }^{* *}$ Qingyu Wu, Professeure d'anglais, lycée $n^{\circ} 9$ de Beihai, Province de Guangsei, Chine. 
Les Sciences de l'éducation - Pour l'Ère nouvelle, vol. 42, n 4, 2009

\section{Introduction}

Depuis le début du XXI' siècle, l'ambition de la Chine est que chacun ${ }^{1}$ ait accès à l'éducation. La première mesure prise en 2000 est la mise en place de l'enseignement primaire universel obligatoire permettant l'élimination de l'analphabétisme : à la fin de l'année 2002, plus de $92 \%$ des enfants étaient scolarisés et $5 \%$ étaient analphabètes ${ }^{2}$. Le deuxième événement marquant concerne l'enseignement supérieur qui atteint désormais un public de masse : en 1998, sept millions d'étudiants étaient inscrits à l'université ou dans d'autres établissements de l'enseignement supérieur; cinq ans plus tard, ils étaient seize millions (Bulletin de l'éducation, UNESCO, 2003).

Le processus de modernisation économique de la Chine réclame un développement très rapide de l'éducation et génère également une forte demande de la part de la population, notamment de la classe moyenne représentant environ deux cents millions de personnes, souhaitant bénéficier d'une meilleure qualité de vie.

Pour autant, ces remarquables avancées cachent des inégalités entre les filles et les garçons du point de vue de l'accès à certaines filières de l'enseignement supérieur. Les filières scientifiques et techniques à l'université restent actuellement masculines à plus de $70 \%$ alors que la proportion de filles $(45,7 \%)$ et de garçons $(54,3 \%)$ à l'université en 2004 tend à se rejoindre 3 . L'augmentation des filles à l'université (en dix ans $+11,25$ points) concerne surtout les domaines des lettres, des langues, des sciences humaines, des sciences sociales et du vivant ${ }^{4}$.

Cette répartition inégale entre les filières selon les sexes des étudiants n'est pas un phénomène propre à la Chine; c'est un phénomène mondial (BAUDELOT \& ESTABLET, 1992).

Toutefois, nous montrerons que contrairement aux autres pays où «les filles accèdent en grand nombre à l'université, elles accroissent leur présence dans toutes les filières, y compris celles où traditionnellement elles n'avaient aucune part $"^{5}$, ce n'est pas le cas actuellement en Chine pour les filières scientifiques et techniques.

En effet, entre 1949 (fondation de la République Populaire de Chine) et 1966 (début de la "révolution culturelle»), les filles se dirigeaient davantage vers les études

1. Afin de ne pas alourdir le texte, nous utiliserons en général le masculin neutre pour parler des étudiants et étudiantes, des filles et des garçons.

2. Bulletin de l'éducation, UNESCO, 2003.

3. Revue de l'éducation chinoise, avril $2005, \mathrm{n}^{\circ} 125$.

4. Revue des étudiants chinois «La richesse et les meilleurs candidats du concours d'entrée à l'université», septembre 2005, n 109.

5. Baudelot C. \& Establet R. Allez les filles. Paris : Seuil, 1992, p. 59. 
scientifiques et techniques qu'actuellement, puisqu'elles représentaient $40,3 \%$ des effectifs de ces filières ${ }^{6}$, alors que la France, par exemple, comptait en $196031 \%$ de filles dans les filières scientifiques des universités (BAUDELOT \& ESTABLET, 1992) et $6 \%$ de filles en classes préparatoires scientifiques de type maths-physique (C. Fontanini, 1999).

En 1985, le taux d'accès à l'université en Chine, selon le Produit National Brut (PNB) par tête, en dollars, était de 1,2 pour les filles et de 3,2 pour les garçons (PNB Chine $=330$ ) ; en France, il était de 30,5 pour les filles et de 29,5 pour les garçons $(\text { PNB France }=12790)^{7}$.

Cet article a donc pour objectif d'examiner les facteurs explicatifs de la moindre orientation des filles vers les études scientifiques et techniques dans l'enseignement supérieur. Nous montrerons tout d'abord que certaines raisons relèvent de l'histoire de l'éducation en Chine, liée aux différents systèmes économiques et politiques qui se sont succédés dans ce pays ces cinquante dernières années. Ensuite, nous mettrons en évidence que, malgré une histoire éducative et politique très différente entre la Chine et des pays occidentaux comme la France, certaines explications sont communes.

\section{Présentation du système éducatif chinois}

L'ancien système de cinq années obligatoires en primaire a été abandonné au milieu des années 1980 pour être remplacé par neuf années obligatoires dont six années de primaire

( 7 à 13 ans) et trois ans de secondaire (13 à 16 ans). Dès le primaire, les élèves ont plusieurs professeurs qui enseignent différentes matières.

Sur les murs des bourgades rurales, en gros caractères, s'affichent les slogans : «Même si on est pauvre, les enfants doivent pouvoir étudier à l'école. Même si la vie est dure, les enfants ne doivent pas en pâtir ${ }^{8}$. Ces slogans officiels cachent une réalité qui est le coût exorbitant de l'école pour beaucoup de Chinois, en particulier dans les régions les plus pauvres du pays. La scolarité à l'école primaire est officiellement gratuite mais les divers frais, comme les livres par exemple, sont à la charge des élèves et sont trop lourds pour les populations rurales, engendrant de nombreux retraits d'enfants de l'école.

Le livre à succès «Le journal de Ma Yan. La vie quotidienne d'une écolière chinoise» présenté par P. Haski (2002) montre bien la misère des familles dans certaines régions et leurs difficultés d'envoyer leurs enfants à l'école.

6. Source web $\mathrm{n}^{\circ} 1$ : www.China.org.cn/education/history/woman and sciences/jdxybd/1949-1966.htm

7. BAUdelot C. \& eSTABLET R. Op. cit., p. 47.

8. Se référer au site suivant : http://www.couleursdechine.org/education chine. php?langue=fr, p. 1. 
Les Sciences de l'éducation - Pour l'Ère nouvelle, vol. 42, n 4, 2009

Les élèves doivent aller au collège le plus proche de leur domicile, selon une carte scolaire établie par l'administration. A la campagne, les collégiens et collégiennes sont souvent internes.

L'entrée au lycée, en général à l'âge de seize ans, est soumise à la réussite à un examen. Les élèves, ayant obtenu de bons résultats, peuvent être admis dans des écoles nationales (école normale, école médicale, lycée d'enseignement général, école technique, école militaire...) qui débouchent sur un emploi de fonctionnaire avec la garantie de l'emploi et un salaire décent. Ceux et celles qui n'ont pas obtenu une moyenne suffisante peuvent s'inscrire dans des écoles privées dont le diplôme a une moindre valeur sur le marché du travail et ne permet pas de devenir fonctionnaire.

L'enseignement au niveau secondaire comporte les mêmes matières que l'enseignement dans les pays occidentaux. Toutefois y est ajoutée une matière "politique» qui consiste à inculquer aux élèves les fondements théoriques du marxisme et du socialisme.

Les lycées d'enseignement général accueillent les meilleurs élèves de l'enseignement secondaire qui sont les principaux lauréats aux concours d'entrée à l'université.

Pour poursuivre ses études en Master et Doctorat, il faut passer un concours. Plus le niveau est élevé, plus le concours est sélectif.

En juin 2007, plus de 9,5 millions de lycéens se sont inscrits au concours d'entrée à l'université. C'est le plus grand nombre de particpants depuis trente ans. Cinq cent mille élèves sont admis directement à l'université sur recommandation. Sur les 9,5 millions de participants, environ 5,6 millions sont retenus. Le concours national est l'un des événements les plus importants dans la vie d'un écolier chinois9.

Dans la constitution chinoise, la loi sur l'enseignement obligatoire et la loi sur la protection des droits et intérêts des femmes explicitement que les femmes doivent bénéficier des mêmes droits à l'éducation que les hommes ${ }^{10}$.

\section{Résultats et orienta- tions scolaires des filles et des garçons}

Comme dans beaucoup de pays (BAUDELOT \& ESTABLET, 1992), en Chine, les résultats des filles sont meilleurs que ceux des garçons à l'école primaire.

Dès le collège, des écarts de résultats entre les disciplines commencent à apparaître. La

9. Source web $\mathrm{n}^{\circ} 8$ : www.cctv.com/program/journal/200706608/106585.shtml (8 juin 2007)

10. Source web $\mathrm{n}^{\circ} 9:$ http://french.cri.cn/cinaabc/chapter12/chapter120503.htm; http://www.new.xinhuanet.com/st/2004-10/19/content-2109463.html; http://www.couleursdechine.org/. 
plupart des filles conservent de bonnes notes en chinois (mandarin), en histoire- géographie, en langue étrangère, en chimie et en biologie. Mais en mathématiques, physique et informatique, les meilleurs résultats sont majoritairement obtenus par des garçons.

Depuis 1997, le taux de réussite au baccalauréat des filles et des garçons est à peu près équivalent (respectivement $86,7 \%$ et $87,2 \%$ en 2005 ).

Au concours d'entrée à l'université, les filles représentent $65 \%$ des meilleurs candidats au concours littéraire et, depuis quelques années, $50 \%$ au concours scientifique ${ }^{11}$. Les meilleurs candidats au concours d'entrée à l'université obtiennent une bourse du gouvernement et des grandes entreprises. Meilleurs sont les résultats des étudiants et étudiantes, plus importante est la somme; celle-ci peut aller jusqu'à l'équivalent de 5000 euros, somme équivalente à cinq ans de frais d'université ${ }^{2}$. Par ailleurs, les étudiants des filières scientifiques et techniques, dans les universités les plus prestigieuses et dans les cycles les plus élevés, ont plus de chances d'obtenir une bourse d'études.

À partir de la classe de première en lycée général, les élèves commencent à choisir leur orientation : classe littéraire (arts, lettres, langues, sciences humaines et sociales) ou classe scientifique (technologies, sciences de la vie et sciences sociales). Après le concours d'entrée à l'université, les jeunes Chinois réitèrent leurs choix qui déterminent ceux de Master et de Doctorat. La spécialité de formation est déterminante dans l'accès à l'emploi, le statut et le salaire. Par ailleurs, le processus permettant de changer de spécialité ou de filière est assez compliqué dans l'université chinoise. En général, les étudiants ne sont pas autorisés à en changer.

L'accès des garçons à l'université reste un peu plus important que celui des filles (respectivement $54,3 \%$ et $45,7 \%$ en 2004 ), notamment dans les campagnes ${ }^{13}$. Depuis 2001, la présence des filles dans les filières langues, lettres, sciences humaines et sociales des universités prestigieuses $(53 \%)$ a dépassé celle des garçons ${ }^{14}$.

Les garçons dominent actuellement les filières scientifiques et techniques du premier au troisième cycle $(70 \%)$.

Il ne faut pas oublier que, de 1949 à 1966, les filles étaient plus présentes qu'actuellement dans les filières scientifiques et techniques (40\%).

Entre 1966 et 1976, le concours d'entrée à l'université a été supprimé et l'enseignement supérieur a été modifié. En 1977, le concours d'entrée à l'université est rétabli et c'est à partir de cette année que la part des filles dans les filières scientifiques et techniques commence à diminuer.

11. Revue de concours d'entrée à l'université chinoise, octobre 2005, nº 79.

12. Revue des étudiants chinois, septembre $2005, \mathrm{n}^{\circ} 109$.

13. Revue de l'éducation chinoise, avril $2005, \mathrm{n}^{\circ} 125$.

14. Revue de concours d'entrée à l'université chinoise, avril 2005, nº 77. 
Les Sciences de l'éducation - Pour l'Ère nouvelle, vol. 42, n 4, 2009

Dans les années 1980, les filles représentent $35 \%$ des effectifs des étudiants dans les filières scientifiques et techniques, puis maintenant $30 \%$.

He Qingjiang (2001), sociologue et professeur de l'université de Pékin, explique que, dans les années 1950-1960, les femmes «osaient» concurrencer les hommes dans les études scientifiques. Pendant la « révolution culturelle» (entre 1966 et 1977), les jeunes chinois des deux sexes ont été envoyés dans les campagnes pour faire changer les mentalités des paysans et paysannes.

Après la "révolution culturelle», la plupart des jeunes retournent vivre en ville. Parmi les personnes restant à la campagne, les femmes sont majoritaires (65\%). Entre 1977 et 1980, les femmes se présentent ainsi moins (38,2 \%) aux concours d'entrée à l'université que les hommes. De plus, le concours scientifique nécessitant plus de temps pour le préparer, les femmes préfèrent passer le concours littéraire à cause de la charge des enfants et du travail domestique.

Après la «révolution culturelle», le courant d'égalité hommes-femmes s'amoindrit et la culture traditionnelle revient progressivement, y compris à l'école.

\section{La diminution des filles dans les filières scientifiques dans l'enseignement supérieur}

Actuellement, les filles représentent environ $40 \%$ des élèves en terminale scientifique. Entre 2000 et 2004, la part des filles dans cette section augmente dans les régions de Pékin et Shanghai (de $39,5 \%$ à $42,6 \%$ ) et dans les sept provinces principales $(38,6 \% \text { à } 41,1 \%)^{15}$.

En 2004, les filles issues des classes scientifiques des lycées des régions de Pékin et Shanghai, se sont orientées pratiquement autant vers la filière "droit économie, gestion" $(34,2 \%)$ que vers la voie «sciences du vivant et de la santé» (35\%) et la filière «sciences et technologies» $(30,8 \%)$.

Pour les sept autres provinces, on constate les mêmes tendances : 33,8 \% pour "droit, économie et gestion», 35,7\% pour «sciences du vivant et de la santé» et $30,4 \%$ pour "sciences et technologies ${ }^{16}$.

Ainsi, seulement un tiers des bachelières scientifiques s'oriente vers les filières scientifiques et techniques à l'université.

Dans les cinq universités prestigieuses (Tsinhua, Pékin, Fudan, Zhejiang et Nanjing), la part des filles dans les filières scientifiques évolue peu depuis 2000. On relève tout de même que, dans les filières "agriculture et industrie chimique» et

15. Revue de concours d'entrée à l'université chinoise, décembre 2004, $\mathrm{n}^{\circ} 72$.

16. Revue de concours d'entrée à l'université chinoise, avril 2005, $\mathrm{n}^{\circ} 77$. 
«informatique» de ces universités, la présence des filles est supérieure à la moyenne des autres disciplines scientifiques.

\begin{tabular}{|c|c|c|c|c|c|c|}
\hline & Informatique & Ecologie & $\begin{array}{c}\text { Physique, } \\
\text { électronique, } \\
\text { génie civil }\end{array}$ & Mathématiques & $\begin{array}{c}\text { Agriculture } \\
\text { industrie } \\
\text { chimique }\end{array}$ & Moyenne \\
\hline 2000 & $29 \%$ & $14,5 \%$ & $20,7 \%$ & $25,2 \%$ & $33,5 \%$ & $25,05 \%$ \\
\hline 2001 & $30,3 \%$ & $15,8 \%$ & $21,8 \%$ & $26,4 \%$ & $34,2 \%$ & $26,1 \%$ \\
\hline 2002 & $31,7 \%$ & $17,4 \%$ & $22,7 \%$ & $27 \%$ & $36,4 \%$ & $27,4 \%$ \\
\hline 2003 & $34,4 \%$ & $19 \%$ & $24,9 \%$ & $28,1 \%$ & $37,6 \%$ & $29 \%$ \\
\hline 2004 & $35,8 \%$ & $20,1 \%$ & $26,3 \%$ & $28,4 \%$ & $37,8 \%$ & $29,9 \%$ \\
\hline
\end{tabular}

Source : Revue de concours d'entrée à l'université chinoise, $\mathrm{n}^{\circ} 77$, avril 2005

Présence des filles dans les cinq universités prestigieuses de 2000 à 2004, cycle Bac +1 .

L'augmentation de la part des filles en informatique peut s'expliquer par le développement récent et très important de ce secteur d'activité en Chine. En France, de la même manière, au début de l'ère informatique (dans les années 1980), les filles y étaient assez bien représentées, ce qui n'est plus le cas vingt ans plus tard car les filles désertent désormais ces études (COLLET, 2006).

La part des filles en écologie, bien qu'augmentant régulièrement, est moindre que pour les garçons. Viennent ensuite la physique, l'électronique et le génie civil. Sontelles considérées par les Chinoises comme des disciplines "plutôt» masculines?

La part des filles en mathématiques a évolué lentement et est proche de la moyenne.

Par ailleurs, la proportion des filles varie selon les régions. Dans l'enseignement supérieur, elles sont plus nombreuses en sciences et technologie dans les provinces de Pékin et Shanghai que dans les provinces du sud-est puis du nord-ouest. De manière générale, elles étudient plus ces disciplines lorsqu'elles habitent dans les villes, notamment à Pékin (capitale politique) et à Shanghai (capitale économique) qu’à la campagne.

Enfin, l'université de Tsinghua, la plus prestigieuse des écoles d'ingénierie, ouverte aux filles depuis seulement 1977, ne compte en 2004 que $22 \%$ de filles (Revue d'étudiants chinois, $\mathrm{n}^{\circ} 109$, septembre 2005)

On a donc vu que, dans l'enseignement supérieur scientifique, le nombre de filles est moins élevé dans le premier cycle que celui des garçons. Ce nombre diminue au fur et à mesure que le cycle est élevé. L'entrée en Master et en Doctorat est conditionnée par les résultats aux concours qui sont organisés par le ministère de l'Éducation chaque année.

Par exemple, le nombre de filles étudiant les mathématiques fondamentales et appliquées dans les quatre universités (Zhjiang, Pékin, Fudan et Nanjing) en Master est assez faible et chute nettement entre le Master et le Doctorat. 
Les Sciences de l'éducation - Pour l'Ère nouvelle, vol. 42, n 4, 2009

\begin{tabular}{|c|c|c|c|c|c|c|c|c|c|c|c|c|c|c|c|c|}
\hline & \multicolumn{4}{|c|}{ Zhejiang } & \multicolumn{4}{|c|}{ Fudan } & \multicolumn{4}{|c|}{ Pékin } & \multicolumn{4}{|c|}{ Nanjing } \\
\hline & \multicolumn{2}{|c|}{ Master } & \multicolumn{2}{|c|}{ Doctorat } & \multicolumn{2}{|c|}{ Master } & \multicolumn{2}{|c|}{ Doctorat } & \multicolumn{2}{|c|}{ Master } & \multicolumn{2}{|c|}{ Doctorat } & \multicolumn{2}{|c|}{ Master } & \multicolumn{2}{|c|}{ Doctorat } \\
\hline & NT & NF & NT & NF & NT & NF & NT & NF & NT & NF & NT & NF & NT & NF & NT & NF \\
\hline 2002 & 70 & 10 & 34 & 3 & 65 & 9 & 35 & 3 & 88 & 12 & 42 & 2 & 68 & 7 & 35 & 1 \\
\hline 2003 & 70 & 9 & 41 & 1 & 80 & 9 & 42 & 2 & 97 & 12 & 53 & 5 & 100 & 11 & 51 & 3 \\
\hline 2004 & 75 & 10 & 49 & 3 & 90 & 13 & 42 & 3 & 101 & 14 & 52 & 2 & 98 & 10 & 34 & 3 \\
\hline
\end{tabular}

Nombre de filles en mathématiques fondamentales et appliquées.

\section{Explications propres à la Chine}

À notre connaissance, très peu de recherches ont été menées en Chine, jusqu'à présent, sur la déperdition des filles dans les filières scientifiques et techniques au fur et à mesure du cursus scolaire. En outre, l'absence d'indicateurs et de collecte de données spécifiques aux femmes, notamment dans le domaine des sciences, ingénierie et technologies, rend difficile les études sur ce sujet ${ }^{17}$.

\subsection{Influence familiale}

Q. Wu (2006) a fait une étude auprès de 801 élèves en classe de terminale scientifique et littéraire ( 420 filles et 381 garçons) de 2 lycées généraux à Pékin $\left(n^{\circ} 3\right.$ et $\left.n^{\circ} 6\right)$ et à Shanghai ( $\mathrm{n}^{\circ} 9$ et $\left.\mathrm{n}^{\circ} 24\right)$ et auprès de 511 étudiants (210 filles et 291 garçons) de première année de Licence (L1) des filières sciences $\&$ technologies et lettres, langues, sciences humaines des universités prestigieuses de Pékin et Fudan pour tenter de comprendre la déperdition des filles dans les filières scientifiques à l'université, notamment en Master et Doctorat.

Près de $50 \%$ des élèves et étudiants de l'enquête estiment que leurs choix d'orientation ont été influencés par leurs parents. C'est plus particulièrement vrai pour les filles.

La Revue des étudiants chinois ${ }^{18}$ a publié un article intitulé «Où sont les meilleures candidates des concours d'entrée à l'université?».

Les propos de ces brillantes étudiantes mettent en évidence que :

17. UNESCO. Priorités pour la science au XXI ${ }^{e}$ siècle en Asie-Pacifique. Conférence UNESCO en AsiePacifique, Sydney, Australie, 1-5 décembre 1998. Résumé du rapport Femmes, sciences, Ingénierie et Technologie. Paris : Editions UNESCO, 1998.

18. La Revue des étudiants chinois, octobre 2002, n 96, p. 17. 


\section{Christine FONTANINI et Qingyu WU}

- les résultats scolaires aux concours ne sont pas un frein à l'orientation scolaire et professionnelle vers les sciences pour les filles.

-certaines d'entre elles abandonnent leur projet initial finalement car, d'une part, elles n'ont pas de soutien de leur entourage (parents, professeurs...) vers cette voie et, d'autre part, la situation du marché du travail et la crainte du chômage les découragent.

En voici quelques extraits ${ }^{19}$ :

La meilleure candidate dans le concours scientifique de Zhejiang, 2001

"Je désirais devenir ingénieur, mais ma maman espérait que je devienne médecin. J'ai hésité beaucoup. Finalement, je me suis orientée vers la médecine. En fait, comme ma maman le dit : le métier de médecin est plus favorable aux filles que celui d'ingénieur; il est relativement stable; il n'y a pas beaucoup de chômage. Le médecin a plus de temps libre et gagne beaucoup d'argent».

La meilleure candidate dans le concours scientifique de Guangdong, 2002

"J'ai hésité longtemps entre gestion informatique de l'université de Tsinghua et commerce international de l'université de Pékin, mais, à la fin, j’ai décidé de choisir commerce international. Les deux spécialités sont très rentables, mais, pour choisir une spécialité masculine et entrer à l'université de Tsinghua, il faut beaucoup de courage. Peut être que je manque de ce courage. J'ai toujours eu envie de choisir ce que je veux, mais...".

La meilleure candidate dans le concours scientifique de Hubei, 2001

"J'aimais l'astronomie dès l'enfance. Mais j'ai choisi ingénierie financière comme orientation universitaire. Il n'y a pas de débouchés en astronomie. De plus, une femme astronome, c'est un peu bizarre pour la plupart des personnes. L'intérêt personnel était fragile face à la réalité. Une bonne spécialité est très importante pour les filles, notamment dans la situation actuelle».

La meilleure candidate dans le concours scientifique de Hubei, 2002

"Je voulais choisir mathématiques appliquées, mais c'était une spécialité masculine. En plus, j'ai douté de mes compétences et j'ai eu peur de ne pas trouver de débouchés dans l'avenir. Par ailleurs, mon professeur n'a pas soutenu mon choix. J'ai eu peur de ne pas trouver de travail après mes études. J'ai alors opté pour la comptabilité que mes parents avaient choisie pour moi».

19. Cités et traduits par WU Q. Rôle de l'orientation dans le choix des filières scientifiques et techniques de l'enseignement supérieur, le cas des filles en Chine. Mémoire de Master 1. Rennes: Université Rennes 2, 2006, pp. 34-35. 
Les Sciences de l'éducation - Pour l'Ère nouvelle, vol. 42, n 4, 2009

Par ailleurs, pour que leurs enfants réussissent au concours d'entrée à l'université, les parents chinois des milieux favorisés demandent souvent à ceux-ci de suivre «le cours spécifique» pendant leur temps libre.

Les parents n'échappent pas toujours aux stéréotypes de sexe. Le cours spécifique proposé par les parents aux filles est plus souvent lié aux lettres/arts et, pour les garçons, lié aux sciences. Autrement dit, par «le cours spécifique», les parents incitent plus les filles à s'intéresser aux lettres/arts et les garçons, aux sciences.

Dès l'école maternelle, le fossé se creuse donc entre les deux sexes : les petites filles commencent à apprendre la peinture, le piano, la calligraphie... et les petits garçons, l'arithmétique, la géométrie, la natation, le tennis de table... Au collège et au lycée, «le cours spécifique» évolue vers "le cours de rattrapage»: pendant les week-end, les jeunes filles suivent des cours de langue étrangère, écriture, musique... et les garçons, des cours de mathématiques, physique, chimie, informatique...

L'étude de Q. Wu (2006) montre que les filles fréquentant le cours de rattrapage «littéraire» réussissent mieux dans les disciplines littéraires que dans les disciplines scientifiques et s’orientent plus vers les études littéraires.

\subsection{La place des chercheurs scientifiques en Chine}

Selon le recensement des scientifiques, la part des femmes dans la recherche, l'ingénierie, l'agronomie et l'aéronautique est de $21 \%$ (YIPING HUO, 1995). Parmi elles, peu y occupent des postes à responsabilités. En 1995, les femmes dans le domaine scientifique et technique représentent $1,04 \%$ des actives occupées et en $2001,1,28 \%{ }^{20}$.

Plus le niveau augmente, moins les femmes sont présentes. A titre d'exemple, en 2003, les académiciennes représentent seulement 5,6\% des effectifs à l'Académie des Industries de Chine et $5 \%$ des effectifs à l'Académie des Sciences de Chine ${ }^{21}$. Cette part féminine n'a d'ailleurs pas évolué depuis 1993 puisqu'elle était déjà de 5,4 \% selon Yiping Huo (1995).

Par ailleurs, il semble que les chercheurs scientifiques chinois travaillent plus que ceux des autres pays dans le monde. Une enquête menée en $2004^{22}$ par Yang Weiping (membre de l'Académie des Sciences de Chine qui est l'institution de recherche la plus grande et la plus renommée) sur les conditions de vie des chercheurs scientifiques montre tout d'abord que les chercheurs scientifiques de Chine travaillent plus par semaine ( 9,68 heures en moyenne) que ceux d'Asie en général ( 9,25 heures), d'Europe

20. Source web $\mathrm{n}^{\circ} 2:$ http://www.sociology.cass.net.cn/shxw/shjgyfc/t20040927 2865.htm

21. Source web $n^{\circ} 3:$ http://www.caspe.ac.cn/elites/elt105.html

22. Source web $n^{\circ} 4:$ http://www.new.xinhuanet.com/st/2004-10/19/content-2109463.html 
Christine FonTANINI et Qingyu Wu

(8,91 heures), d'Australie (8,33 heures), d'Amérique du Nord (8,67 heures) et d'autres pays divers ( 9 heures). D'autre part, les chercheurs chinois ont moins de temps de loisirs (faire du sport, aller au cinéma, théâtre, restaurant...) que les chercheurs des autres pays du monde. Ces conditions de travail des chercheur-e-s expliquent sans doute en partie que la Chine se place au cinquième rang mondial de publications (Sciences Citation index cité par B. Belloc \& L. Roos, 2006). La progression des publications est spectaculaire et continue de croître de façon exponentielle : elle a doublé en six ans.

Ainsi, la vie des chercheurs scientifiques chinois apparaît peu diversifiée et le travail occupe la plupart de leur temps.

Les femmes scientifiques ont a priori le même rythme de travail que leurs collègues masculins mais doivent aussi assurer les charges familiales (travail domestique, éducation de-s enfant-s). Elles peuvent rencontrer des difficultés pour assumer ce triple rôle : mère, épouse et exerçant une profession où les hommes sont majoritaires.

Une des premières mesures prises après la mort de Mao Zedong en 1976, a été la réorganisation de la très officielle Fédération des femmes, dissoute pendant la « révolution culturelle». Certaines de ces déclarations montrent le retour en force de la famille : «les femmes travailleuses, les membres des communes, les techniciennes et scientifiques doivent travailler et étudier dur; mais elles doivent aussi consacrer une part importante de leur vie à s'occuper de leur maison et de leurs enfants ${ }^{23}$.

On peut donc penser que les conditions actuelles du métier de chercheur scientifique en Chine peuvent rebuter les jeunes filles à s'y engager, lesquelles font alors des «choix raisonnables» (DURU BELLAT, 1990) en se détournant de ces filières.

\section{En Chine... comme ailleurs...}

\subsection{Exclusion pendant très longtemps des femmes des savoirs...}

En Chine, comme dans les autres pays du monde, pendant très longtemps, les filles ont été exclues des pouvoirs et des savoirs. Elles ont souffert d'une éducation rituelle pendant environ 2000 ans.

L'éducation des filles commence ainsi : «Quand une fille naissait, on la plaçait sous le lit : signe de la soumission absolue; on lui donnait pour jouer, une tuile,

23. Cité par M. Rodière : Rodière M. Chine. Peut-on parler d'amour? In : PAQUOT E. (dir.). Terre des Femmes. Paris : La Découverte, 1982, p. 184. 
Les Sciences de l'éducation - Pour l'Ère nouvelle, vol. 42, n 4, 2009

symbole des travaux ménagers qu'on exigerait d'elle plus tard.» (Lu Shan, 921, «Livre d'histoire de Han ") ${ }^{24}$. La femme doit être toujours craintive et travailler jour et nuit. Elle n'a pas droit aux louanges, mais, par contre, doit accepter sans murmures tous les blâmes.

Dans la tradition chinoise, le mari est le Ciel de sa femme. Personne ne pourrait échapper au Ciel, donc aucune femme ne doit quitter son époux. Le Ciel est unique, donc le mari aussi. Autrement dit, la femme ne doit avoir durant sa vie qu'un seul mari; la femme, par elle-même, n'existe pas; son seul chemin est la soumission.

L'acquisition des savoirs est le privilège de l'homme. Seulement une petite partie des filles d'origine favorisée peut acquérir une instruction en art : musique, poésie, calligraphie et dessin. Cette minorité de filles est envoyée ensuite au Palais Impérial et servira l'Empereur. L'Empereur est le fils de Ciel. Il possède le pouvoir le plus sacré et les meilleures femmes (jolies, élevées et obéissantes). Quant aux savoirs savants (la logique, la géométrie, l'arithmétique, etc.), ils sont absolument interdits aux filles car ils sont le symbole de la sagesse et des pouvoirs de l'homme. L'accès aux savoirs savants incarne l'intelligence et la place de l'homme au-dessus de la femme.

L'éducation est déterminée par l'ordre social et l'assure à son tour. Donc, l'éducation des filles a pour objectif de restreindre le développement intellectuel des femmes et de les former à devenir de bonnes mères de famille et de bonnes épouses.

Le gouvernement de la République de Chine a bouleversé, dès 1949, la place sociale des femmes tant du point de vue juridique, politique, économique, culturel, social et familial (YIPING HuO, 1995).

De plus, depuis 1993, selon Yiping Huo (1995), «le gouvernement et les écoles encouragent et soutiennent à différents niveaux les filles voulant se consacrer aux sciences, notamment aux mathématiques et aux sciences expérimentales, et s'efforcent de créer pour elles des conditions favorables leur permettant de mettre pleinement en valeur leurs compétences et leurs talents et de concurrencer ainsi les garçons ${ }^{25}$.

Toutefois, comme le dit N. Mosconi (1994) : «Aujourd'hui, les hommes continuent à se réserver en partie un certain nombre de savoirs, avec les pouvoirs qui leur sont liés.

La séparation des sexes entre les filières n'obéit plus à un modèle mécanique mais statistique. Il y a des filières et des domaines de savoirs, où les minorités de filles sont

24. Cité et traduit par WU Q. Rôle de l'orientation dans le choix des filières scientifiques et techniques de l'enseignement supérieur, le cas des filles en Chine. Mémoire de Master 1. Rennes: Université Rennes 2, 2006, p. 40.

25. HUO Y. L'enseignement scientifique des filles en Chine. In : CLAIR R. (dir.). La formation scientifique des filles : un enseignement au-dessus de tout soupçon? Paris : Unesco, 1995, p. 103. 
vues comme des exceptions, c'est-à-dire comme n'entamant pas la règle qui veut que les filles en restent éloignées ou en soient écartées.

Ces filières et ces savoirs que les hommes entendent se réserver, ce sont les filières scientifiques et techniques, tous ces savoirs "prométhéens» qui, dans notre société, peuvent se transformer en compétences, c'est-à-dire en un savoir qui fonde un pouvoir. [...] Ainsi, en s'assurant le monopole des formations scientifiques et techniques, ainsi que de la plupart des niveaux les plus élevés des formations supérieures, les hommes s'assurent le monopole sur les positions d'ingénieurs et sur les positions de cadres supérieurs dans les entreprises privées et publiques comme dans la haute administration, ainsi que sur les fonctions politiques.

Autrement dit, c'est toujours par le maintien d'un quasi-monopole sur un certain nombre de savoirs que le groupe des hommes s'assure un monopole sur les pouvoirs essentiels dans notre société26"

\subsection{Le rôle de l'institution scolaire}

Le système scolaire chinois, comme ceux des autres pays, transmet une image des femmes largement nourrie des conceptions masculines, c'est-à-dire une image qui laisse aux hommes le monopole des idéaux collectifs capables de nourrir des projets existentiels importants et qui laisse aux femmes les projets "modestes» que suppose leur statut de dominées.

De nombreuses études nord-américaines et européennes (citées entre autres par N. Mosconi, 1994) ont montré que le nombre d'interactions entre les enseignants des deux sexes et les garçons en classe est plus important que le nombre d'interactions avec les filles. Les garçons reçoivent plus d'attention que les filles dans les classes des sciences.

Ces interactions peuvent être des questions adressées aux élèves, le fait de leur donner la parole, mais aussi des rétroactions sur les prestations orales ou écrites, des corrections, des évaluations, félicitations ou critiques...

De plus, la nature des questions adressées aux filles et aux garçons diffère. Les professeurs posent aux garçons plus de questions complexes, semi-ouvertes ou ouvertes qu'aux filles.

Une enquête effectuée dans quatre lycées généraux à Shanghai en 1994 par Anne Garance-Primel (1995) montre que les professeurs attribuent plus de qualités positives aux filles qu'aux garçons et que les filles exercent mieux le métier d'élève que les

26. MosConi N. Femmes et savoirs. Paris : L'Harmattan, 1994, p. 217. 
Les Sciences de l'éducation - Pour l'Ère nouvelle, vol. 42, n 4, 2009

garçons. Mais «lorsqu'on compare les qualités reconnues aux garçons et aux filles, celles attribuées aux garçons sont généralement considérées comme plus valorisantes, ainsi les garçons sont plus intelligents ( $13 \%$ contre $3,5 \%$ pour les filles) et plus adroits (6,5\% contre $2 \%$ pour les filles); les filles sont plus travailleuses ( $12 \%$ contre $8,5 \%$ pour les garçons) et plus sérieuses ( $10,5 \%$ contre $3 \%$ pour les garçons). Les filles sont plus incapables ou stupides que les garçons $»^{27}$.

Comme le souligne cette chercheuse : «ces portraits féminins et masculins ne sont pas spécifiquement chinois; le fait que ces clichés soient très semblables à ceux existant dans le monde occidental est plus qu'inquiétant».

En effet, en 1990, M. Duru-Bellat (1990) avançait que «les garçons sont souvent considérés comme "sous réalisateurs» : ils sont intelligents, ils ont des possibilités indéniables, mais ils ne les exploitent pas entièrement, faute de faire les «efforts» suffisants. Aux filles, au contraire, les professeurs n'ont pas tendance à attribuer des ressources intellectuelles inemployées. Les filles «font ce qu'elles peuvent» : elles n'ont pas de grandes capacités intellectuelles, mais elles réussissent grâce à leur travail $\aleph^{28}$.

Q. Wu (2006) montre que généralement les garçons ont plus confiance en leurs aptitudes dans les disciplines scientifiques. Ils choisissent donc plus souvent que les filles les filières scientifiques et des universités prestigieuses.

Quelques études faites en Europe sur les manuels scolaires (citées entre autres par N. Mosconi, 1994) ont permis de montrer les stéréotypes sexistes qui y étaient présents et l'usage que les enseignant-e-s en font (peu de remise en cause des stéréotypes).

D'après Q. Wu (2006), au cours de la longue civilisation chinoise, les femmes ont influencé le cours de l'histoire du pays. Néanmoins, dans les manuels d'histoire chinois, elles sont souvent négligées, ou dans l'ombre de la gloire de leurs maris, ou jugées comme impertinentes.

Par exemple, Wu Zetian, est la seule impératrice régnante de l'histoire de Chine (dynastie des Tangs). Pendant son règne (près d'un demi siècle), la Chine fut prospère et puissante, sa population stable, son armée repoussa plusieurs fois des invasions étrangères. Mais, on retient en général plus d'elle, qu'elle était ambitieuse et cruelle ${ }^{29}$.

Dans l'historiographie scolaire chinoise, aussi, on vante plus les grands hommes. Les grandes femmes doivent céder devant la culture héroïque : pas de grande savante,

27. GARANCE-PRIMEL A. Le regard des enseignants chinois sur les élèves : un avantage trompeur pour les filles. In : ClAIR R. (dir.). La formation scientifique des filles : un enseignement au-dessus de tout soupçon? Paris : Unesco, 1995, p. 110.

28. DuRU-BeLlat M. L'école des filles. Quelles formations pour quels rôles sociaux? Paris : L'Harmattan, 1990 , p. 65.

29. Source web $n^{\circ} 5:$ http://french.cri.cn/chinaabc/chapter16/chapter160309.htm 
Christine Fontanini et Qingyu Wu

pas de grande femme politique. On préfère admirer le rôle de mère : elles sont source de bien-être pour leurs enfants, elles n'ont ni vie affective autonome, ni vie sociale, ni intérêt personnel, et elles suivent volontairement ce que leurs maris font.

\section{Le double rôle d'enseignant chinois : professeur et orienteur}

Il n'y pas de conseiller d'orientation psychologue dans le système scolaire chinois. C'est le rôle des professeurs.

Par ailleurs, dans l'éducation traditionnelle chinoise, les professeurs non seulement transmettent les savoirs aux élèves mais aussi «forment leurs personnalités».

Aujourd'hui, les élèves chinois conservent la tradition de respecter leurs professeurs et de suivre leurs conseils. L'enquête de Q. Wu (2006) montre que les filles répondent plus à l'attente des professeurs que les garçons.

Les professeurs jouent donc un rôle important vis-à-vis des filles et notamment vers leur orientation vers des études et carrières scientifiques.

Cependant, en Chine comme ailleurs, les professeurs ou conseiller-e-s d'orientation n'évitent pas les stéréotypes de sexe dans les conseils qu'ils donnent aux élèves... Les sciences et technologies sont considérées comme un domaine masculin. Ils encouragent donc plus les garçons à s'engager dans ce type d'études et les filles, vers des carrières littéraires.

\section{L'apprentissage des rôles dans la famille}

La socialisation commence dès la naissance. Les parents ont des représentations bien précises souvent marquées par les stéréotypes de sexe sur ce qu'est et doit être un garçon ou une fille. En conséquence, ils ne se comportent pas de la même manière avec une fille ou un garçon et les éduquent différemment : les filles sont ainsi invitées à une douce passivité et à une certaine dépendance par rapport aux adultes, tandis que les garçons sont poussés à l'autonomie, à l'activité et à l'indépendance.

Dans la famille se transmettent des règles et modèles, tant par inculcation des modes de pensée, de sentir et d'agir, que par intériorisation inconsciente à travers l'imitation des personnes.

Selon Q. Wu (2006), dans la société chinoise, la réussite sociale (professionnelle et financière) d'un homme lui apportera souvent ce qu'il veut, mais pour une femme, elle perdra souvent ce qu'elle veut (par exemple : un bon mariage). 
Les Sciences de l'éducation - Pour l'Ère nouvelle, vol. 42, n 4, 2009

Une enquête menée par l'université de Shanghai en 2002 (citée par Q. Wu, 2006) montre que la participation aux tâches domestiques varie selon le sexe et la région : les filles font plus de travaux domestiques que les garçons en général mais en plus, les filles des campagnes font plus de travaux domestiques que celles des villes.

Bien que les adolescents et adolescentes chinois fassent de moins en moins de travaux domestiques car les parents gâtent de plus en plus leur enfant (surtout en ville, la plupart des familles chinoises ont un enfant unique), les tâches domestiques restent plus l'apanage des filles... comme en France d'ailleurs (INSEE, les jeunes, 2000).

De même, dans ces deux pays, les activités de loisirs des filles sont moins diversifiées que celles des garçons et se situent plus dans l'univers domestique ${ }^{30}$.

Pour la lecture, $62 \%$ des filles chinoises entre 14 ans et 20 ans aiment les romans d'amour et lisent des magazines de mode, de beauté, de cuisine, etc. Les garçons préfèrent les romans de science-fiction et d'aventures et lisent des revues spécialisées dans le sport, moto, jeux vidéo, etc.

Quand elles regardent la télévision, les filles préfèrent les programmes traitant de relations humaines et des sentiments alors que les garçons préfèrent les programmes traitant de relations politiques et scientifiques.

Pour la consultation sur Internet, les goûts des filles et des garçons sont assez différents. Les filles préfèrent "bavarder», s'écouter et se donner la parole mutuellement et les garçons, jouer aux jeux d'aventure ou de compétition.

En somme, les filles recherchent plus «le caractère coopératif entre les individus» et les garçons, la compétition et le pouvoir.

\section{Le marché du travail}

Les filles réussissent souvent mieux que les garçons, mais leur réussite scolaire ne se transforme pas encore en réussite sociale.

Le diplôme des filles est moins rentable que celui des garçons sur le marché du travail chinois, notamment depuis l'expansion de l'enseignement supérieur en 1997. Le chômage des diplômés du supérieur concerne plus souvent les filles ${ }^{31}$. D'après $\mathrm{Q}$. Wu (2006), «Les filles souhaitent chercher un travail et les garçons souhaitent chercher un meilleur travail $»^{32}$.

30. Source web $n^{\circ} 6$ : www.sina.edu.cn. Voir également : GALANT C. (dir.). Les jeunes. Portrait social. Paris : INSEE, 2000, (collection contours et caractères).

31. Source web $n^{\circ} 7:$ http://www.51job.com.cn/arts-sciences-job/diaocha/conten-2004.html

32. WU Q. Rôle de l'orientation dans le choix des filières scientifiques et techniques de l'enseignement supérieur, le cas des filles en Chine. Mémoire de Master 1, Université Rennes 2, 2006, p. 66. 


\section{Christine FONTANINI et Qingyu Wu}

Selon Yiping Huo (1995), depuis l'ouverture de la Chine vers une économie de marché, les entreprises sont plus autonomes pour recruter leur personnel et ont tendance à recruter plus des hommes que des femmes, "arguant du fait que ces dernières seront rapidement confrontées à des problèmes réels comme le mariage et la maternité» 33 .

Ainsi, certains employeurs préfèrent embaucher des hommes ou des femmes qui ont déjà leur enfant. Cette situation est accentuée chez les filles dans les filières scientifiques et techniques.

Les métiers scientifiques et techniques sont proposés davantage aux hommes en Chine. De plus, les filles qui ont choisi les filières scientifiques et techniques se confrontent à plus d'obstacles (préjugé social, force physique, mariage, maternité...) que celles qui choisissent les autres filières sur le marché du travail.

Comme le souligne E. M. Rathgeber (1996) : «Dans tous les pays, la nécessité pour les femmes d'allier leurs responsabilités professionnelles et familiales nuit aux perspectives d'emploi des femmes scientifiques. Les employeurs pensent souvent que l'engagement des femmes envers la science est moins soutenu que celui de leurs collègues masculins, surtout si elles sont mariées et ont une famille. Cela peut avoir un effet, non seulement en ce qui concerne leur décision d'engager une scientifique mais sur le genre de travail qui lui sera attribué $»^{34}$.

\section{L'impact des médias}

Aujourd'hui, les médias jouent un rôle important dans le processus de socialisation des adolescent-e-s (PASQUIER, 1999). Les valeurs transmises par certaines séries de télévision (par exemple, rôles traditionnels des hommes et des femmes) peuvent conditionner les choix scolaires et/ou professionnels des adolescent-e-s.

Le message des médias en Chine est uniforme : le destin de la femme est le mariage et la beauté; le destin de l'homme, l'argent et le pouvoir.

Une étude menée en octobre 2004 par la revue chinoise "Télé et moi »35 sur les publicités dans CCTV (Chine) montre que la présence des hommes et des femmes à l'écran correspond à certains produits :

33. Huo Y. L'enseignement scientifique des filles en Chine. In : ClAIR R. (dir.). La formation scientifique des filles : un enseignement au-dessus de tout soupçon? Paris : Unesco, 1995, p. 155.

34. Rathgeber E. M. Pourquoi l'éducation? Possibilités d'éducation et perspectives de carrière des femmes dans la science, la technologie et le génie. In : Groupe sur l'égalité des sexes. L'Autre développement - l'égalité des sexes en sciences et technologie. Commission des sciences et de la technique au service du développement des Nations Unies, 1996, http://idrc.ca/fr/ev-29523-201-16DO TOPIC. html, p. 12.

35. Télé et moi, 2006, $\mathrm{n}^{\circ} 44$, cité par Q. Wu. 
Les Sciences de l'éducation - Pour l'Ère nouvelle, vol. 42, n 4, 2009

- articles de toilette, pour bébés et produits ménagers pour le sexe féminin;

- voitures, produits électroniques (notamment portable, ordinateur...) et boissons alcooliques pour le sexe masculin.

Les femmes occupent principalement dans les publicités ces trois professions : professeur, infirmière et secrétaire et les hommes ingénieur, médecin et patron.

La presse ne fait pas exception aux tendances générales. Les femmes ne peuvent "se réaliser» que dans l'amour, en étant objet du désir masculin, mais pas moins accessoirement femme d'intérieur.

La plupart des romans d'amour proposés aux adolescentes chinoises répètent toujours ce conte ancien : le héros sauve la beauté de différentes façons : un homme de type "superman" (beaucoup d'argent, grands pouvoirs et très intelligent) rencontre une femme "pauvre» (très jolie, soumise et d'origine modeste), il est fasciné par la beauté de cette femme, et puis ils s'aiment. Ainsi, grâce à l'amour de l'homme, la femme devient une «superwoman" (bonne mère et bonne épouse).

A travers tous ces médias, ce sont des modèles de comportement, des manières d'être et d'agir, conforme à tel ou tel sexe, qui sont proposés aux adolescents et adolescentes. L'uniformité massive des modèles proposés par les médias, si sécurisante soit-elle, n'offre aux jeunes comme seule alternative que l'acceptation ou le rejet en bloc.

\section{Conclusion}

Malgré les progrès scolaires incontestables des filles dans l'enseignement supérieur en Chine, il convient de rappeler que ces avancées cachent de fortes inégalités entre les filles des villes et les filles de la campagne (comme d'ailleurs entre les garçons des deux milieux mais dans une moindre mesure) : en 2003, les urbaines représentent 45,5\% des sortants de l'enseignement supérieur (niveau Bac + 3), et les rurales $32 \%$ (Wen Dongmao, 2004). C'est à partir du lycée que les filles des campagnes commencent à «disparaître» du système scolaire.

La plupart des sociologues chinois expliquent la faible présence des filles rurales dans l'enseignement supérieur par l'histoire de l'éducation des filles et par le manque de moyens financiers des parents pour financer des études.

En ville, les parents n'ont, en général, qu'un seul enfant, ce qui leur permet d'investir plus facilement dans son éducation. À la campagne, les familles ont souvent davantage qu'un enfant. En outre, leurs revenus sont souvent plus faibles qu'en ville.

Ces deux dernières décennies, la plupart des fonds gouvernementaux pour aider les étudiants ont été distribués selon leurs résultats et non selon leur situation financière. Le gouvernement chinois a décidé pour la rentrée 2007 d'aider ceux et celles qui 
n'ont pas les moyens de payer leurs frais d'études avec des prêts ou des bourses notamment pour les étudiants pauvres qui ont de bons résultats ${ }^{36}$.

D’après He Qinglian (2001), la pauvreté renforce l'inégalité des sexes. Si une famille peut envoyer un enfant à l'université, elle choisira un fils plutôt qu'une fille. Ce choix s'explique par le fait que ce sont les fils qui assurent la subsistance à long terme de la famille. Le caractère patrilocal du mariage dans la Chine rurale signifie que le fils après son mariage continue en principe de vivre avec ses parents et leur apporte une sécurité financière, alors que la fille mariée quitte sa famille pour aller vivre avec celle de son mari.

La société chinoise est toujours marquée par une préférence pour la descendance mâle et par une discrimination à l'égard des filles avant même leur naissance : avortement sélectif, surmortalité féminine excessive dans l'enfance mais aussi à l'âge adulte, abandon majoritairement de filles... (ATTANÉ, 2006).

Par ailleurs, la modernisation économique, en creusant des inégalités déjà patentes, n'a fait qu'envenimer la situation, notamment pour les femmes : plus de chômage que pour les hommes, perception de salaires largement inférieurs à ceux des hommes pour un travail et une qualification identiques...

«Revers de la libéralisation économique et sociale dans ce pays marqué par trois décennies de communisme pur et dur, les rapports de pouvoir traditionnels, irrépressivement défavorables aux femmes, ont resurgi. Leur statut millénaire, soumis à l'autorité des hommes, est, dans la société chinoise actuelle, renforcé par une vision peu propice de leur rôle dans la mondialisation, et dans toutes les nouvelles rivalités qu' elle engendre ${ }^{37} »$.

36. www.chine-nouvelle.com, 20/07/07.

37. Attane I. Les "femmes manquantes» en Asie. In : Ockrent C. (dir.). Le livre noir de la condition des femmes. Paris : XO Éditions, 2006, p. 31. 
Les Sciences de l'éducation - Pour l'Ére nouvelle, vol. 42, n 4, 2009

\section{Bibliographie}

ATTANe I. Les «femmes manquantes» en Asie. In : OCKRENT C. (dir.). Le livre noir de la condition des femmes. Paris : XO Éditions, 2006, pp. 30-51.

Baudelot C. \& Establet R. Allez les filles. Paris : Seuil, 1992.

BELLOC B. \& ROOS L. La recherche scientifique chinoise: organisation, situation et tendances. Ambassade de France en Chine, Service pour la science et la technologie, 27 février 2006.

Chongguo C. Chine : l'envers de la puissance. Paris : Mango, 2006.

COLLET I. L'informatique a-t-elle un sexe? Paris : L'Harmattan, 2006.

CURTIN K. Les femmes dans la révolution chinoise. Paris : Éditions la Brèche, 1975.

DURU BELLAT M. L'école des filles. Quelles formations pour quels rôles sociaux? Paris : L'Harmattan, 1990.

FONTANINI C. Les filles face aux classes de mathématiques supérieures et spéciales: Analyse des déterminants des choix d'une filière considérée comme atypique à leur sexe. Thèse de Doctorat en Sciences de l'Éducation. Dijon : Université de Bourgogne, 1999.

GARANCE-PRIMEL A. Le regard des enseignants chinois sur les élèves : un avantage trompeur pour les filles. In : CLAIR R. (dir.). La formation scientifique des filles : un enseignement au-dessus de tout soupçon? Paris : Unesco, 1995, pp. 109-112.

HASKI P. Le journal de Ma Yan. La vie quotidienne d'une écolière chinoise. Paris : Ramsay, 2002.

Huo Y. La recherche en histoire de l'éducation chinoise en Chine. Histoire de l'éducation, janvier 1994, n 61, pp. 41-59.

Huo Y. L'enseignement scientifique des filles en Chine. In : CLAIR R. (dir.). La formation scientifique des filles : un enseignement au-dessus de tout soupçon? Paris : Unesco, 1995, pp. 101-108.

Huo Y. Le lycée en Chine (1922-2002). Histoire de l'éducation, janvier 2004, n 101 , pp. 67-85.

Galant C. (dir.). Les jeunes. Portrait social. Paris : INSEE, 2000, (collection contours et caractères). 
Christine FonTANINI et Qingyu Wu

MosCONI N. Femmes et savoirs. Paris : L'Harmattan, 1994.

PASQUIER D. La culture des sentiments. L'expérience télévisuelle des adolescents. Paris : Éditions de la Maison des sciences de l'homme, 1999.

QINGLIAN H. Analyse du changement de la place des femmes chinoises aujourd'hui. Revue de recherche sur la Chine moderne, octobre 2001.

Rathgeber E. M. Pourquoi l'éducation? Possibilités d'éducation et perspectives de carrière des femmes dans la science, la technologie et le genie. In : Groupe sur l'égalité des sexes. L'Autre développement - l'égalité des sexes en sciences et technologie. Commission des sciences et de la technique au service du développement des Nations Unies, 1996.

Rodière M. Chine. Peut-on parler d'amour? In : PAQuOt E. (dir.). Terre des femmes. Paris : La Découverte, 1982.

UNESCO. Bulletin de l'éducation. Paris : Éditions UNESCO, 2003.

UnESCO. Priorités pour la science au XXI ${ }^{e}$ siècle en Asie-Pacifique. Conférence UNESCO en Asie-Pacifique, Sydney, Australie, 1-5 décembre 1998. Résumé du rapport Femmes, sciences, Ingénierie et Technologie. Paris : Editions UNESCO, 1998.

WEN D. A comparative study on the gender differences of higher education opportunity, school work achievement and graduate employement in China. New York : University Press, 2004.

Wu Q. Rôle de l'orientation dans le choix des filières scientifiques et techniques de l'enseignement supérieur, le cas des filles en Chine. Mémoire de Master 1. Rennes : Université Rennes 2, 2006.

\section{Revues}

Revue des étudiants chinois, "Où sont les meilleures candidates des concours d'entrée à l'université?», Ministère de l'éducation, octobre 2002, n 96.

Revue des étudiants chinois, Ministère de l'éducation, mars 2004, nº 108.

Revue des étudiants chinois, "La richesse et les meilleurs candidats du concours d'entrée à l'université", Ministère de l'éducation, septembre 2005, nº 109.

Revue de concours d'entrée à l'université chinoise, Ministère de l'éducation, décembre $2004, n^{\circ} 72$. 
Les Sciences de l'éducation - Pour l'Ère nouvelle, vol. 42, n 4, 2009

Revue de concours d'entrée à l'université chinoise, Ministère de l'éducation, avril 2005, $\mathrm{n}^{\circ} 77$.

Revue de concours d'entrée à l'université chinoise, Ministère de l'éducation, octobre $2005, \mathrm{n}^{\circ} 79$.

Revue de l'éducation chinoise, Ministère de l'éducation, avril 2005, nº 125.

\section{Webographie}

Source web $\mathrm{n}^{\circ} 1$ : www.China.org.cn/education/history/woman and sciences /jdxybd/1949-1966.htm

Source web $n^{\circ} 2$ :

http://www.sociology.cass.net.cn/shxw/shjgyfc/t20040927 2865.htm

Source web $n^{\circ} 3$ : http://www.caspe.ac.cn/elites/elt105.html

Source web $\mathrm{n}^{\circ} 4$ : http://www.new.xinhuanet.com/st/2004-10/19/content2109463.html

Source web $\mathrm{n}^{\circ} 5$ : http://french.cri.cn/chinaabc/chapter16/chapter160309.htm

Source web $n^{\circ} 6$ : http : www.sina.edu.cn

Source web $\mathrm{n}^{\circ} 7$ : http://www.51job.com.cn/arts-sciences-job/diaocha/conten2004.html

Source web $n^{\circ} 8$ : www.cctv.com/program/journal/200706608/106585.shtml(8 juin 2007)

Source web $\mathrm{n}^{\circ} 9:$ http://french.cri.cn/cinaabc/chapter12/chapter $120503 . \mathrm{htm}$

http://www.new.xinhuanet.com/st/2004-10/19/content-2109463.html ; http://www.couleursdechine.org/ 


\title{
The place of the girls in the scientific higher education in China : one step forward, two steps back.
}

\begin{abstract}
For ten years, the number of students both male and female has dramatically increased in China. For all that, this «massification» of the higher education does not entail equality between the girls' and the boys' access to the various university fields. Thus, we notice, that with the end of the communist system, advocating equality between both sexes in the professional sphere and with the opening of China to the market economy, women and men tend to opt for studies and professions similar to those opted for by their homologues in the occidental world, namely a massive orientation of the girls towards the literary fields, the human and social sciences... and the boys, towards sciences and technics.
\end{abstract}

Key Words : Sciences. Orientation. Gender. China.

\section{El lugar de las muchachas en la enseñanza superior cientifica en China : un paso para adelante, dos para atrás}

Resumen : Desde hace diez años, el número de estudiantes aumenta considerablemente en China. Por eso, esta «massification » de la enseńanza superior no tiene como consecuencia, muy al contrario, una igualdad entre las chicas y los chicos en el acceso a las diferentes hileras universitarias.

Así, comprobamos, que con fin del sistema comunista, preconizando una igualdad entre ambos sexos en la esfera profesional y con la abertura de China a la economía de mercado, las mujeres y los hombres tiende a elegir estudios y profesionales semejantes a sus colegas del mundo occidental, a saber una orientación maciza de las chicas hacia las hileras literarias, las ciencias humanas y sociales... y los chicos, las ciencias y las técnicas.

Palabras claves : Ciencias. Orientación. Género. China. 